\title{
THEORETICAL METHODS FOR THE ANALYSIS OF SPECTRA OF HIGHLY VIBRATIONALLY EXCITED POLYATOMIC MOLECULES
}

\author{
F. BORONDO
}

Dep. de Química, C-XIV, Universidad Autónoma de Madrid, Cantoblanco-28049 Madrid (Spain)

\section{J. M. GOMEZ LLORENTE}

Dep. de Física Fundamental y Experimental, Universidad de La Laguna, 38203 Tenerife

(Spain)

\section{R. M. BENITO}

Dep. de Física Aplicada, E.T.S.I. de Telecomunicación, Universidad Politécnica de Madrid, 28040 Madrid (Spain)

(Received 17 June 1991; in final form 15 October 1991)

\begin{abstract}
The vibrational spectra of classically chaotic systems are usually very complicated and seemingly unassignable. In this paper, two methods for the analysis of spectra of highly vibrationally excited polyatomic molecules are described, and some results for the floppy molecules HCN and LiNC are presented. By application of these methods, relevant information on the underlying dynamics is obtained, thus establishing a bridge between the spectroscopy and the dynamics of these systems.
\end{abstract}

KEY WORDS: Vibrorotational states, Molecular Dynamics, Molecular Spectra.

\section{INTRODUCTION}

Recent years have witnessed an extraordinarily increasing interest in the dynamics of vibrationally high excited molecules. The reasons that have contributed to such renewed interest are numerous, but mainly due to the necessity of a general knowledge of the dynamical processes that may occur at high vibrational energies, in order to gain quantitative and general understanding of intramolecular processes such as isomerization and intramolecular vibrational relaxation, unimolecular reactive processes such as unimolecular decomposition and photodissociation, and collisional reactive processes such as those taking place through activated complexes.

Most intramolecular processes, i.e. isomerization, require the vibrational excitation of the molecule in order for them to occur. The excitation may be accomplished in a particular way, in principle, by selecting a specific mode to put the energy in. However, nothing assures us that this energy will remain in the chosen mode. Intramolecular energy transfer may occur leading to relaxation and energy randomization. This randomization is in fact the main hypothesis behind statistical theories $^{1}$ for unimolecular processes such as the RRKM theory which has been successfully applied to many cases. However, the existence of cases with non-statistical 
behavior is also well documented, ${ }^{1}$ and this enhances the interest for a better knowledge of the energy transfer mechanisms in highly excited molecules.

All comments stated in the above paragraph are also applicable to unimolecular decomposition processes requiring a previous vibrational excitation of the molecule. In the case of photodissociation, the molecule, through interaction with radiation, changes electronic state. A packet is then formed, that closely corresponds to the initial vibrational state, which evolves on the excited electronic surface towards dissociation. The energy distribution in the different internal degrees of freedom of the fragments will strongly depend on intramolecular dynamical processes.

Most non-direct reactive collisional processes occur via unstable complexes that appear at the so-called transition state. ${ }^{2}$ Such unstable complexes are usually vibrationally very excited species, and their intramolecular dynamics may well determine the distribution of energy among the different degrees of freedom of the products.

The need for a general understanding of the dynamics of molecules in highly lying vibrational states, in order to gain insight into all these processes has encouraged the development of new experimental techniques up to a stage that no one could have predicted a few years ago. Here we will focus our attention on the spectroscopic techniques and mention some of the most frequent application.

\section{EXPERIMENTAL TECHNIQUES}

There is a large group of experimental techniques that use laser pulses in order to first bring up the molecule from the ground vibrational state to a vibrationally high energy region, and then proceed to measure usual spectra, such as IR absorption spectra, IR fluorescence spectra, Raman scattering spectra, etc. Several procedures can be used to increase the vibrational energy of a molecule. For instance, by exciting the molecule to an upper electronic state, it is possible to populate highly lying vibrational states of the upper surface, provided that the equilibrium geometries of the molecule in both electronic states are markedly different. Non adiabatic couplings between both electronic states may lead to internal conversion, i.e. to transitions from the populated vibrational states of the upper surface to the vibrational states of the lower surface with the same total (electronic plus vibrational) energy. Thus, high vibrational levels of the ground electronic state can be populated through internal conversion.

A different technique, that takes advantage of the anharmonicity of potential surfaces, is overtone excitation in the visible or near IR spectral region. Very sensitive methods have been developed to detect the usually weak overtone signals. Finally we will mention the use of multiphoton excitation with IR radiation as another method to populate highly lying vibrational states. We address the reader to Ref. 3 for a detailed discussion on these techniques and further references.

A second group of spectroscopical methods has a slightly different spirit. Here, one starts by creating a localized wave packet whose energy center lies in a vibrationally high energy region of the electronic surface. The packet will evolve on 
this surface producing an energy resolved spectrum with dynamical information. Stimulated-emission pumping (SEP) spectroscopy pertains to this group. ${ }^{4}$ In a typical SEP experiment, a first fixed-frequency laser (pump laser) excites the system from the lowest vibrational state of the ground electronic hypersurface to an assignable vibrational state of an excited electronic state. Deexcitation is then stimulated by a variable frequency laser (dump laser), and transitions occur to the different vibrational states of the lower surface which lie in the Frank-Condon region corresponding to the initial state (see Figure 1a). Thus, markedly different equilibrium geometries for both surfaces will enable the probing of high vibrational states by SEP methods.

Electron photodetachment is another technique pertaining to this second group that have been applied to the spectroscopic study of the transition state region of some reactive processes. ${ }^{5}$ This technique may be applied whenever there exists a stable negative ion with the same composition and similar geometry as the transition state of the neutral reactive system. The electron of the anion is then photodetached by a fixed frequency laser pulse and the kinetic energy of the fragments is analyzed by time-of-flight spectroscopy (see Figure 1b).

By means of these, and other experimental methods, the high energy spectroscopy of many molecules has been and is being studied. We will center our attention in the case of small molecules (3-4 atoms), although most conclusions have a general validity. Some examples of high energy spectra for small polyatomic molecules are the photodissociation spectrum of $\mathrm{H}_{3}^{+}{ }^{6}$ and the SEP spectra of acetylene, ${ }^{7} \mathrm{Na}_{3},{ }^{8}$ $\mathrm{O}_{3}{ }^{9}$ and $\mathrm{HCN} .{ }^{10}$ In most cases the resulting spectra are very congested and seemingly unsassignable by means of the usual spectroscopic methods. Thus, new methods are required to analyze these complicated spectra. Some statistical analysis ${ }^{11}$ of the level spacing and intensity distributions are usually in good agreement with predictions based on random matrix theory. These results have been linked to the mostly chaotic nature of the classical motion at the energies probed experimentally. ${ }^{12}$ Another interesting feature of these spectra is the appearance of regularities after either coarse

(a)
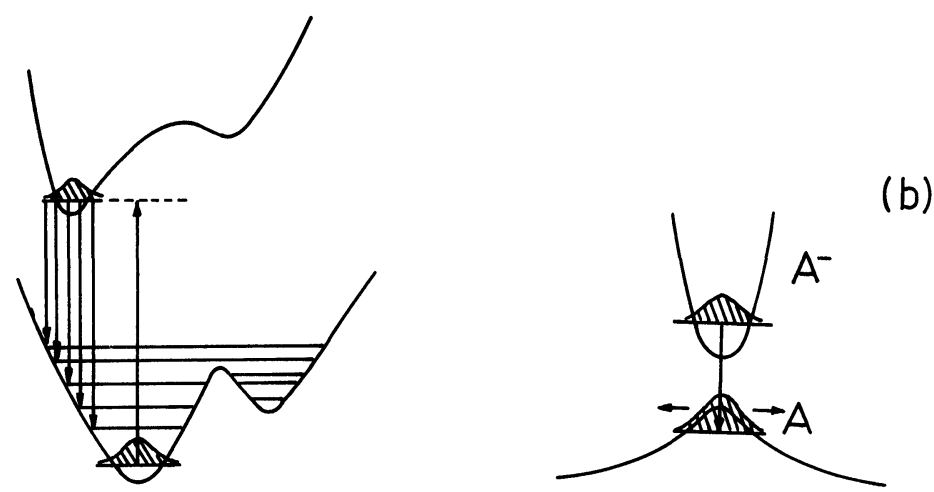

Figure 1 Schematic representation of the processes involved in: (a) SEP spectroscopy, and (b) electron photodetachment spectroscopy. 
graining or Fourier transform. For some systems the high resolution spectra do not always look chaotic, even when chaos seems to dominate most of the classical phase space. This is the case, as it will be seen later, of the SEP spectrum for the HCN molecule.

\section{THEORETICAL METHODS}

In order to understand all these spectral features associated with high vibrational states, new theoretical methods have to be developed. The progress in computational methods allow now the calculation of quantum eigenvalues and eigenfunctions for those vibrational states involved in the experimental spectra of small molecules. ${ }^{13}$ The theoretical simulation of the experiments is therefore possible, but it does not add any new information apart from that concerning the quality of the potential surface. Rather, the most interesting aspect of these quantum calculations is the possibility of studying systematically the structure of high energy wavefunctions. A random nodal pattern behavior appears as the main characteristic of those wavefunctions. This behaviour of the wavefunctions has also been associated with the chaos dominating the classical motion. However and interestingly, individual wavefunctions may be found that present localization over certain regions that correspond closely to phase space structures such as stable islands ${ }^{14}$ or even unstable periodic orbits. ${ }^{15}$ There is strong evidence that the localization is also related to the structures observed in the spectra. ${ }^{16,17}$ Contrarily, the quantum mechanics of certain systems seems to behave as if the classical motion, being chaotic, was regular. This effect is called quantum suppression of chaos. In general, the quantum chaology linked to classical chaos seems to appear at energies always higher than that corresponding to the onset of chaos in classical motion. The aim behind any experimental or theoretical approach is to extract the dynamical information underlying all these observed behaviors, i.e. to relate all observed features in spectra, wavefunction and energy eigenvalues to motions in the system. In order to achieve this goal we should obviously use the language of classical mechanics, assuming that a quantum classical correspondence exists which allows a classical interpretation.

The correspondence between classical and quantum mechanics is well established for globally integrable systems by means of semiclassical mechanics. The classical motion for these systems is confined in phase space to $N$-dimensional tori, $N$ being the number of degrees of freedom. ${ }^{18}$ Each torus can be labeled by a set of $N$ numbers for the values of the $N$ actions, which are global constants of the motion-they have the same functional form of coordinates and momenta everywhere in the phase space of bound motion. All these tori are topologically equivalent and form a continuous family. The semiclassical EBK quantization rule

$$
\int_{C_{i}} \vec{P} \cdot d \vec{q}=h\left(n_{j}+\frac{\alpha_{j}}{4}\right) \quad n_{j}=0,1,2, \ldots
$$

select a discrete set of such tori as quantizable tori. ${ }^{19}$ The action integral in Eq. (1) should be computed along the $N$ topologically independent circuits, $C_{j}$, defining the 
torus. The quantities $\alpha_{j}$ are the Maslov indices, which represent the phase loss at the caustics of the torus; in general $\alpha_{j}$ is equal to 2 for vibrations and 0 for rotations.

The energies of the quantized tori define the semiclassical eigenvalues of the system, and usually are in good agreement with the exact eigenvalues. Quantum eigenfunctions have large amplitude on the configuration space region corresponding to the quantizable tori.

From the practical point of view the methods for semiclassical quantization are very useful in the study of the high lying vibrational states of polyatomic molecules that we are considering in this paper. For such high states the variational and perturbation methods usually used for the approximate solution of the quantum mechanical wave equation are no longer effective. The number of elements required for a basis set, and the number of oscillations in the wavefunction of each state, are too large. However, it is in these conditions where the applicability of the semiclassical theory is more justified, constituting a complementary approach to the traditional methods of quantum mechanics for the study of highly excited molecular vibrations.

Several methods have been proposed in the literature to solve Eq. (1) using a variety of different strategies: numerical construction of the invariant tori ${ }^{20}$ iterative solution of the Hamilton-Jacobi equation for the action function, ${ }^{21}$ classical perturbation theory, ${ }^{22}$ classical variational procedures, ${ }^{23}$ Fourier analysis of the trajectories, ${ }^{24}$ etc. The computational effort implied by these methods increases drastically with the number of degrees of freedom of the system; even some of them are impractical for $N>2$.

An alternative method that maintains the computational efficiency as $N$ increases is that of adiabatic switching. This method is based in an idea of Ehrenfest, who proposed the use of adiabtic conservation of the action to quantize nonseparable Hamiltonian systems. ${ }^{25}$ The first numerical application was done by Solov'ev, ${ }^{26}$ and later Skodje, Borondo and Reinhardt characterized the method in detail, and applied it to a wide variety of coupled oscillators systems. ${ }^{27}$ Several reviews have been published in the literature. ${ }^{28}$

The adiabatic theorem of classical mechanics asserts, roughly speaking, that when a bound, one dimensional system is perturbed slowly enough, the action of the corresponding trajectories is conserved. This result can be used to quantize trajectories, if we write our system Hamiltonian in the form:

$$
H=H_{0}+H_{I}
$$

where $H_{0}$ is a separable approximation to $H$, and $H_{I}$ a correction that doesn't need to be small. Then, we construct the following time dependent Hamiltonian

$$
H(t)=H_{0}+\lambda(t) H_{I}
$$

where $\lambda(t)$ is a smooth switching function going from 0 to 1 in a time $T$. If we now run trajectories with initial conditions on the quantizing torus for $H_{0}$, the actions will be conserved along the switching process, and the final energies will correspond to the EBK quantization of $H$. The root mean deviation can be used as an indicator of the non-adiabaticities encountered in the calculation, and a bound to the error for the semiclassical eigenvalues. 
The adiabatic switching has also proven to be able of obtaining numerically the angle variables of a given problem, ${ }^{29}$ by approximating the instantaneous frequency values by a finite difference formulae, using data from neighbor trajectories. In another publication, ${ }^{30}$ it was shown how to use the adiabatic switching method to generate semiclassical wavefunctions using a coherent superposition of wavepackets, and propagating the trajectories at the center of each packet from $H_{0}$ to $H$. Finally, in Ref. 31 the adiabatic switching method was applied to locate quantized, periodic orbits in bound and reactive multidimensional systems, specially in relation with the "periodic orbits dividing surfaces" and "resonant periodic orbits", ${ }^{32}$ which have been demonstrated to be very important in certain aspects of bimolecular reactions.

Molecular systems are usually globally nonintegrable systems; i.e. there are not $N$ global actions. They may still exist tori in the phase space of the bound motion, but their topology will change from region to region. ${ }^{33}$ In the regions where tori exist, the bound motion is said to be regular. Classical chaos develops at the boundary (separatrix) between two or more such regions, where it may also coexist with islands of regular motion around stable periodic orbits. Wherever chaos sets in, no $N$-dimensional tori can be found. The only possibility is the existence of $(N-m$ )-dimensional tori, $m$ being a positive integer (when $m=N-1$ the tori degenerate into periodic orbits). The volume occupied by the chaotic regions grows with the strength of the non-linearities (i.e. increasing the energy) without any constraint except for the constancy of some global integrals of motion such as the energy, the angular momentum, etc.

Chaos may dominate the classical motion but the phase space can still be highly structured. Reduced-dimensionality tori (periodic orbits included) always exist and it is well documented their influence in the dynamics of atomic and molecular systems. ${ }^{34,14,16,35,36,37}$ The periodic orbit semiclassical theory based on Gutzwiller's summation formula ${ }^{38}$ has been applied to simple two degrees of freedom systems ${ }^{34}$ to calculate the density of states, but dynamical information is not readily obtained from these semiclassical theories.

Here we will review a series of methods recently developed ${ }^{35,36}$ to extract dynamical information from either experimental or theoretical spectra of vibrationally excited molecular systems, whose internal classical motion is partially or totally chaotic. In first place, we will present a methodology which starts with the classical simulation of the quantum correlation functions and spectral densities that can be obtained from the experimental spectrum or from a quantum calculation. This first step shows the extent of the quantum-classical correspondence. If this correspondence can be established, the dynamics can be readily extracted by analyzing the classical trajectories used in the simulation. The contributing trajectories also contain information on the phase space structures causing the determinant motions. Quantum effects may also be readily detected in a way that makes easier their explanation.

A second methodology that uses a phase space representation of the quantum wavefunctions will also be presented. These methods are specially useful to analyze the apparently complicated structure of the wavefunctions for high vibrational states. There is no unique way to transform quantum wavefunction into phase space quasiprobability distributions, being each one of these associated to a given 
quantization map. The two distributions more widely used in the literature are the Wigner transform and the Husimi function. The first one was introduced by Wigner in 1932 as a mean to calculate quantum corrections to equilibrium thermodynamical properties, and is given (in one dimension) by:

$$
W(P, q)=\frac{1}{h} \int d \eta e^{i P \eta} \psi\left(p-\frac{\eta}{2}\right) \psi^{*}\left(q+\frac{\eta}{2}\right)
$$

The Husimi function or coherent state representation

$$
H(P, q)=\frac{1}{h}\left|\int d x \exp \left[-\alpha(x-q)^{2}+i P x\right] \psi(x)\right|^{2}
$$

can be shown to be the result of smoothing the Wigner transform with a Gaussian weighting function.

These two functions have several interesting properties in connection with its interpretation as probability densities in phase space. The Wigner transform yield the correct marginal probability distributions $|\psi(q)|^{2}$ and $|\psi(p)|^{2}$; however it can be negative. On the other hand, the Husimi function is always positive definite, since it is the result of a smoothing process of the Wigner function, but it doesn't give the correct marginal probabilities. From a practical point of view, all those problems are not too severe, since both distributions gives usually the same qualitative information. Projections of $W$ or $H$ onto suitable planes are the quantum counterpart of the classical Poincare surface of sections (PSOS). These are the bidimensional representation resulting from the intersection of a given trajectory with an arbitrary plane, and constitute a valuable tool for examining the phase space structure and dynamics of Hamiltonian systems.

To illustrate these methods we have chosen the HCN and LiCN molecules as test cases. For the HCN system, experimental SEP spectroscopic data have been recently obtained in the high energy region. ${ }^{10} \mathrm{~A}$ great amount of theoretical work has also been carried out for this system, both classically and quantally. Our results for the $\mathrm{HCN}$ system have been presented elsewhere. ${ }^{39,40,41} \mathrm{We}$ will condense them here to illustrate the methods that we will then apply to the LiCN system, for which no experimental spectrum has been obtained so far, but for which quantum eigenfunctions have been calculated up to very high energies. ${ }^{42,43}$ The choice of these two systems is also justified by the floppiness of their classical motion at high energies. This fact, together with the possibility of isomerization ( $\mathrm{HNC}$ and LiNC) also at high energies, make these molecules prototypical systems for studying the interplay between the isomerization process and the transition to chaos. Some differences are however expected in the behavior of both systems, since it is known that LiCN presents a phase space much more dominated by chaotic motion than $\mathrm{HCN}$.

The vibrational Hamiltonian $(J=0)$ corresponding to these systems has the following form in scattering coordinates ( $\hbar$ is set to 1 throughout):

$$
H=\frac{P_{R}^{2}}{2 \mu_{1}}+\frac{P_{r}^{2}}{2 \mu_{2}}+\frac{1}{2}\left(\frac{1}{\mu_{1} R^{2}}+\frac{1}{\mu_{2} r^{2}}\right) P_{\theta}^{2}+V(R, r, \theta)
$$


In this equation, $\mu_{1}$ is the $X-\mathrm{CN}$ reduced mass $(X=\mathrm{H}, \mathrm{Li}), \mu_{2}$ the $\mathrm{CN}$ reduced mass, $R$ the distance from $X$ to the center of mass of $\mathrm{CN}, r$ the $\mathrm{CN}$ distance, and $\theta$ is the angle between the $\mathrm{CN}$ axis and the $X$-to-CN vector. The potential energy surfaces, $V(R, r, \theta)$, for these systems are known to a high degree of accuracy. ${ }^{44,45}$ For the HCN/HNC system Murrell et al. reported an analytical expression, which included two and three bodies terms, and whose parameters were determined by minimizing the difference between the observed and calculated vibrational frequencies by a variational method. For the $\mathrm{LiNC} / \mathrm{LiCN}$ system Brocks et al. fitted a Legendre expansion to a set of realistic "ab initio" SCF data ${ }^{45}$. A contour plot of both potential energy surfaces is shown in Figure 2.

Both systems present two stable isomers in the linear configurations, being the $\mathrm{HCN}$ and $\mathrm{LiNC}$ the most stable ones. The barriers for isomerization are relatively modest: $12098 \mathrm{~cm}^{-1}$ for $\mathrm{HCN} / \mathrm{HNC}$ and $3454 \mathrm{~cm}^{-1}$ for $\mathrm{LiNC} / \mathrm{LiCN}$, which causes that the $\mathrm{H}$ and $\mathrm{Li}$ atoms can "orbit" easily around the $\mathrm{CN}$ fragment.
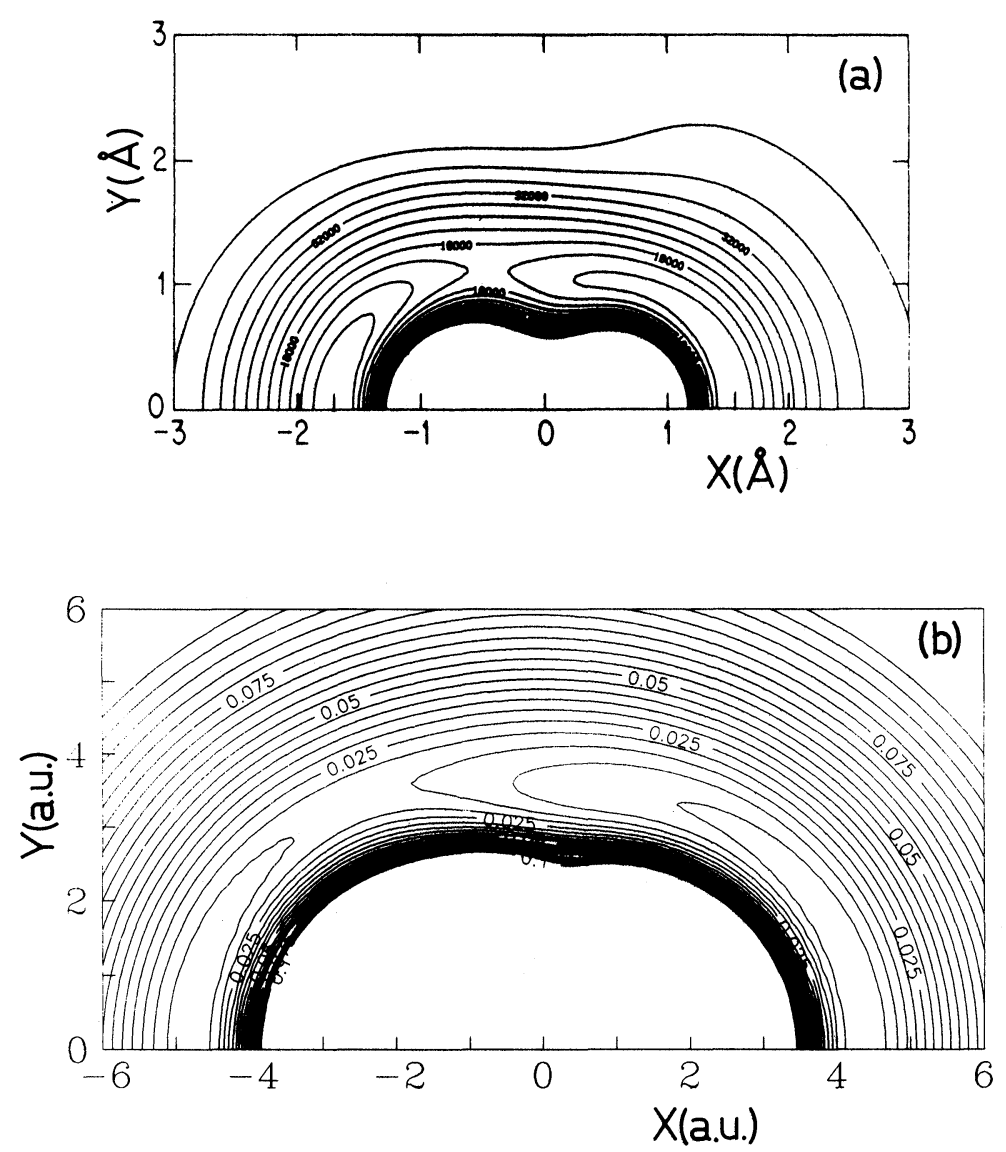

Figure 2 Contour plot of the potential energy surfaces of: (a) HCN/HNC system of Ref. 44, and (b) $\mathrm{LiNC} / \mathrm{LiCN}$ system of Ref. 45. 


\section{SPECTRAL ANALYSIS OF HCN AND LiCN}

The spectra obtained by applying experimental techniques such as SEP, dispersed fluorescence or electron photodetachment can be theoretically written, within the framework of the Frank-Condon approximation, in terms of the vibrational wavefunction for the initial state $|i\rangle$,

$$
I(E)=\sum_{n}|\langle i \mid n\rangle|^{2} \delta\left(E-E_{n}\right)
$$

In this expression $|n\rangle$ is the final vibrational wavefunction of the ground electronic surface to which transitions take place, and $E_{n}$ the corresponding eigenvalue for the energy. The squared magnitude of the Fourier transform (to time domain) of the spectrum

$$
C_{q}(t)=\left|\int \mathrm{e}^{-i E t} I(E) \mathrm{d} t\right|^{2}
$$

Gives the survival probability also known as the quantum correlation function, for it can be written as

$$
C_{q}(t)=\left|\left\langle i\left|\mathrm{e}^{-i H t}\right| i\right\rangle\right|^{2}
$$

A classical analog for the quantum survival probability is given by the equation

$$
C_{c}(t)=\operatorname{Tr}\{\rho(0) \rho(t)\}
$$

where $\rho(0)$ is a phase space representation of the initial quantum distribution $|i\rangle\langle i|$, and $\rho(t)$ is the classical Liouville evolution of $\rho(0)$. The trace $\operatorname{Tr}$ represents an integral over all internal coordinates and momenta of the nuclei. The Fourier transform of the correlation functions (to frequency domain) gives the spectral densities $S_{q}$ and $S_{c}$

$$
S_{q(, c)}=\int \mathrm{e}^{-i \omega t} C_{q,(c)}(t) \mathrm{d} t
$$

The ability of classical mechanics to simulate quantum correlation functions and spectral densities has been repeatedly demonstrated in several cases. ${ }^{46,14,35,36,39,40,47,48}$ To illustrate the methodology, we have chosen the HCN system.

Quite a lot of spectroscopical information is now available for the high vibrational states of the $\mathrm{HCN}$ molecule. Baronavski ${ }^{49}$ studied low resolution dispersed fluorescence spectra for the transition (excited) $\mathrm{A}^{1} \mathrm{~A}^{\prime \prime}$-state $\rightarrow$ (ground) $\mathrm{X}^{1} \Sigma^{+}$-state. The vibrational levels populated in the ground state were mainly those with excitation in the bending and $\mathrm{CN}$ stretching modes. Smith et al. ${ }^{50}$ have obtained infrared overtone spectra with up to six quanta in the $\mathrm{CH}$ stretch mode. The SEP spectrum was reported by Yang et al., ${ }^{10} 80 \%$ of all spectral lines up to $19000 \mathrm{~cm}^{-1}$ were fitted to a normal mode based energy level expression that was first used by these authors to fit their data. Like the fluorescence spectrum, the SEP spectrum sampled a region of phase space with bend and $\mathrm{CN}$ stretch excitations.

A great deal of theoretical work has also been focused on the classical and quantum mechanics of this system. The SEP spectra for both HCN and HNC systems with initial vibrational states of the upper electronic surface successively excited in the bend quantum numbers, $(0, v, 0)$, have been calculated by Bentley et al. ${ }^{51}$ from the 
quantum eigenfunctions and eigenvalues, which they obtained by using the RRGM and DVR-DGB methods, for total angular momentum $J=0$. The empirical potential surface of Ref. 44 was used for the ground electronic state. Let us mention here that the theoretical spectrum and assignments for the $(0,0,0) \mathrm{HCN}$ initial state agree qualitatively well with the experimental SEP spectrum of Ref. 51 . The quantum correlation function corresponding to the calculated spectrum from the $(0,0,0)$ initial state is given in Figure 3a.

Classical trajectory studies ${ }^{52}$ revealed that chaos develops mainly through excitation of the bending mode for both isomers, while stretching excitations do not
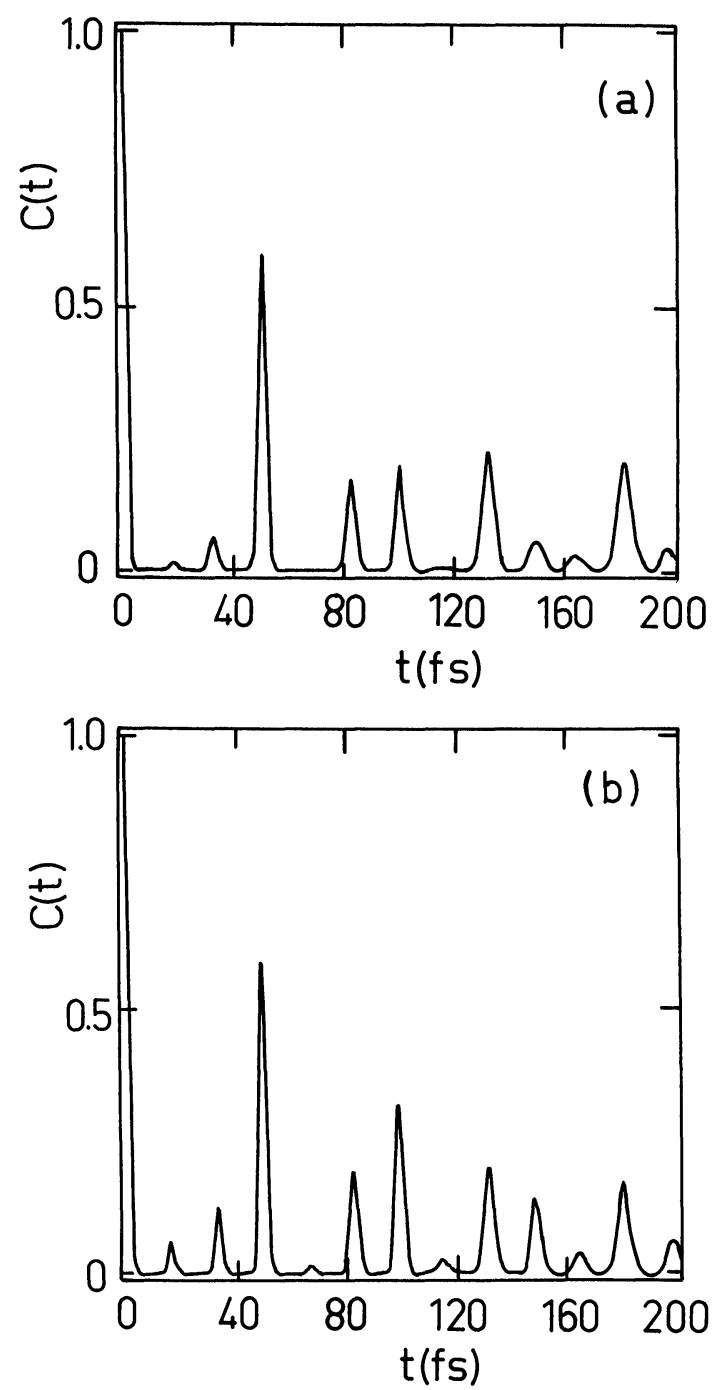

Figure 3 (a) Quantum and (b) classical correlation functions for the $A^{1} A^{\prime \prime} \rightarrow X^{1} \Sigma^{+}$transition of $\mathrm{HCN}$. 
lead easily to chaos, the motion remaining regular even above the first dissociation channel to $\mathrm{CN}+\mathrm{H}$. We may conclude that at the energies probed by the SEP spectrum the proportion of chaotic and regular phase space regions is quite similar, in agreement with the level repulsion detected in statistical analysis of eigenvalues. However, both theoretical and experimental SEP spectra look quite regular. To understand this fact we calculated the classical correlation function defined in equation (10) by running 500 trajectories randomly selected according with the distribution $\rho(0)$. The potential surface and initial state used were those of Ref. 44 (the same surface was used by Bentley et al.). Quantum mechanically, the eigenfunctions corresponding to $J=0$ are defined for values of the angle $\theta \in[0, \pi]$, and they are symmetric with respect to the line $\theta=0, \pi$. This symmetry is imposed classically by reflecting the classical trajectories that reach the symmetry line. This is equivalent to imposing the right symmetry to the distribution $\rho$. The results of these computations are shown in Figure $3 \mathrm{~b}$. This is a clear example of the ability of classical mechanics to reproduce the main features of high energy vibrational spectra. The next step was obviously the analysis of the trajectories that are contributing to the observed structures.

Power spectrum analysis of the trajectories used in the simulation showed that most of them were regular. Surface of section of the same trajectories revealed that most of them were selected from a phase space region covered by tori whose bend and $\mathrm{CN}$-stretch frequencies are in almost 3:1 resonance. In this region a stable periodic orbit was even found that satisfied exactly the $3: 1$ resonance condition, which is presented in Figure 4. However the size of the stable island around this orbit was so small that no influence of it was expected either in the spectrum nor in the form of periodic orbit localization in the wavefunctions. This theoretical analysis

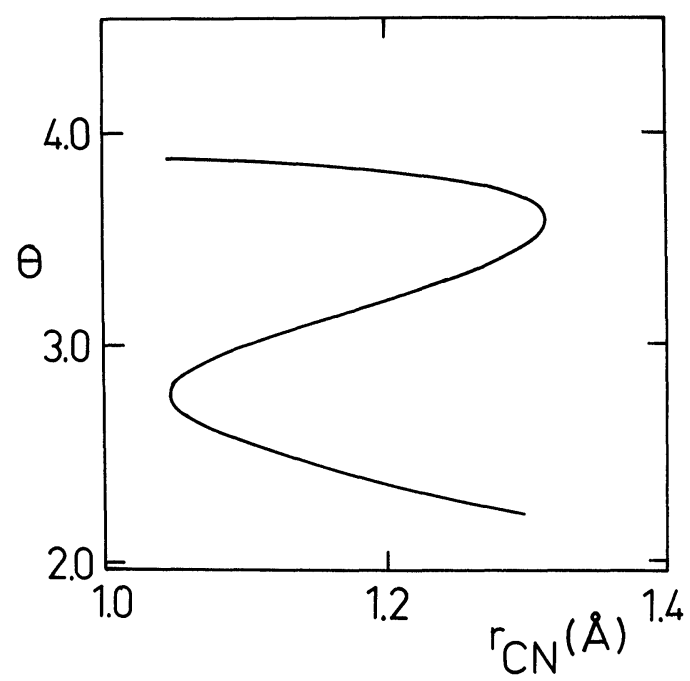

Figure 4 A representative periodic orbit of the $3: 1$ family responsible for the characteristics exhibited by $C(t)$ in Figure 3 . 
is completely consistent with the SEP experimental results, as was discussed in Ref. 41. Other spectra from different initial state and different isomer were also calculated and analyzed by this methodology.

The HCN results illustrate the use of classical spectral analysis in a system for which experimental, as well as quantum theoretical results, were available. For the LiCN molecule no experimental, high-energy, vibrational spectra have been reported so far. Quantum wavefunctions were obtained ${ }^{42}$ up to very high energies (above the isomerization barrier), for a two degrees-of-freedom model in which the $\mathrm{CN}$ bond length was frozen at its equilibrium value. However no SEP spectrum was simulated since information about the upper electronic state is not available at present. Since we are not constrained by any experimental result, and the main aspect in which we are interested is the understanding of the intramolecular dynamics of the system, we proceeded, in the spirit of the SEP spectroscopy, to calculate quantum and classical correlation functions for arbitrary initial wavepackets. The potential surface used in this calculations is that of Ref. 45 , i.e. the same two dimensional surface used to obtain the quantum eigenfunctions and eigenvalues. ${ }^{42}$

The initial wavepacket $|i\rangle$ has a coordinate representation, $\Phi_{i}$, given by the expression

$$
\Phi_{i}=(2 \pi)^{1 / 2}\left(\alpha_{R} \alpha_{\theta}\right)^{1 / 4} \exp \left[-\alpha_{R}\left(R-R^{0}\right)^{2}-\alpha_{\theta}\left(\theta-\theta^{0}\right)^{2}\right]
$$

and for the example that we will present here, it was centered at $R^{0}=3.32 \mathrm{a}$.u. and $\theta^{\circ}=1.5 \mathrm{rad}$; the rest of parameters in Eq. (12) were given the values $\alpha_{R}=3.2 \cdot 10^{-3}$ a.u. ${ }^{-2}$ and $\alpha_{\theta}=6.5 \cdot 10^{-4} \mathrm{rad}^{-2}$. The stick spectrum was then calculated using Eq. (7) and it is presented in Figure 5. This spectrum looks much more complicated than that obtained by Bentley et al. for the HCN (Figure 1 of Ref. 51). There is no hope, we believe, to fit most individual lines of Figure 5 to a simple expression, as was done by Yang et al. ${ }^{10}$ for the HCN system; at least we were not able to do so. However, dynamical information can still be extracted by calculating the survival probability corresponding to this spectrum and its classical analog by means of equations (9) and (10). 1200 trajectories were used to converge the classical correlation function, and the results are shown in Figure 6. Up to $t=700 \mathrm{fs}$ the agreement between quantum and classical results is quite satisfactory. At longer times the classical correlation decays faster than its quantum analog, which also shows additional structure. The relatively fast decay of the classical results has its origin in the chaotic nature of most trajectories used to compute $C_{c}$. More dynamical information can be obtained in frequency domain by calculating the spectral densities by Fourier transforming $C_{q}$ and $C_{c}$. These spectral densities are presented in Figure 7 after convolution with a Lorenztian of width $2 \cdot 10^{-4} \mathrm{a}$.u. This is done to smooth out structure coming from longer times, in which we are not interested here. This finer structure would appear at higher resolution specially in $S_{q}$ and it is due to quantum effects such as tunneling (both dynamical and through the isomerization barrier). At this resolution level both spectral densities agree quite well. An approximate $4: 1$ ratio between stretch and bend frequencies can be observed in the results of Figures 6 and 7. We further investigated this structure by analyzing the 


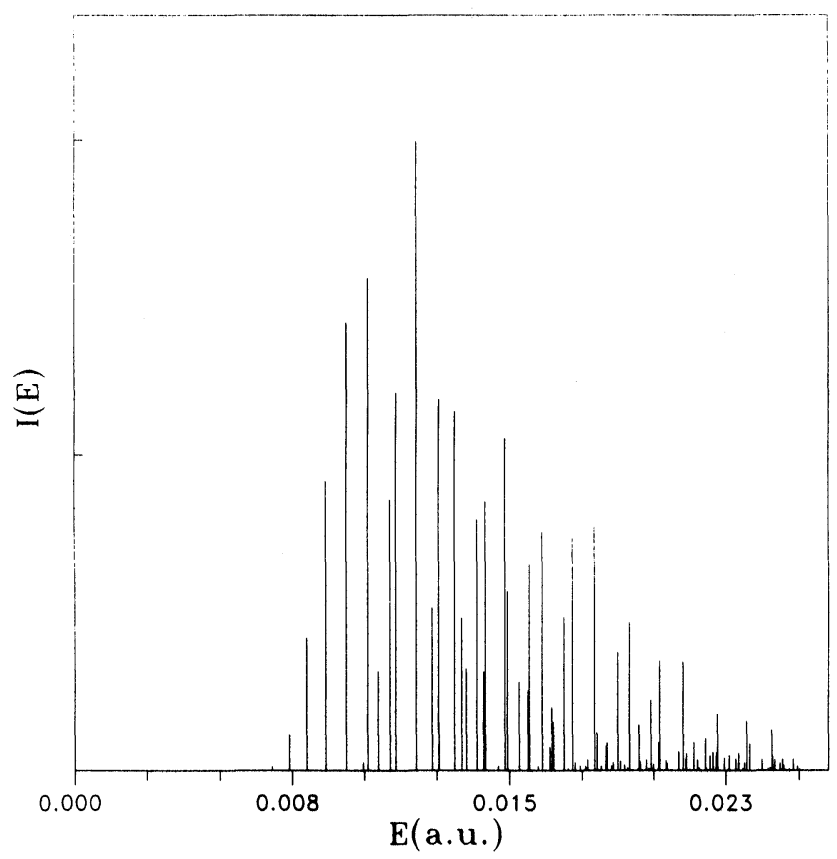

Figure 5 Stick spectrum for the LiNC molecule.

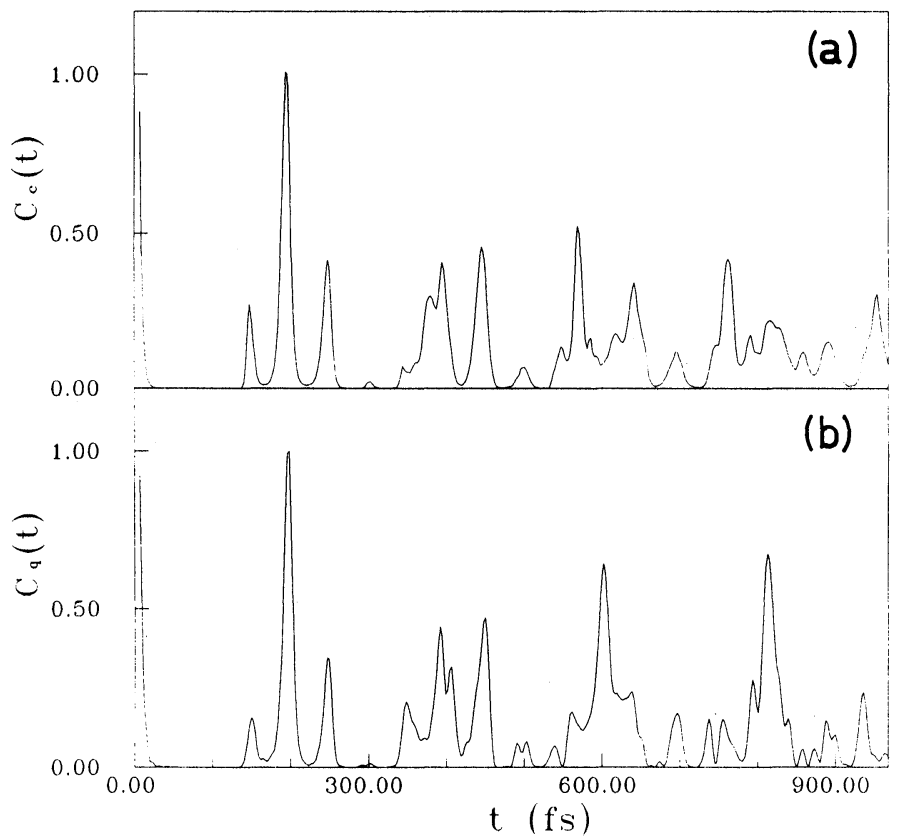

Figure 6 (a) Classical and (b) quantum correlation functions associated to the stick spectrum of Figure 5. 


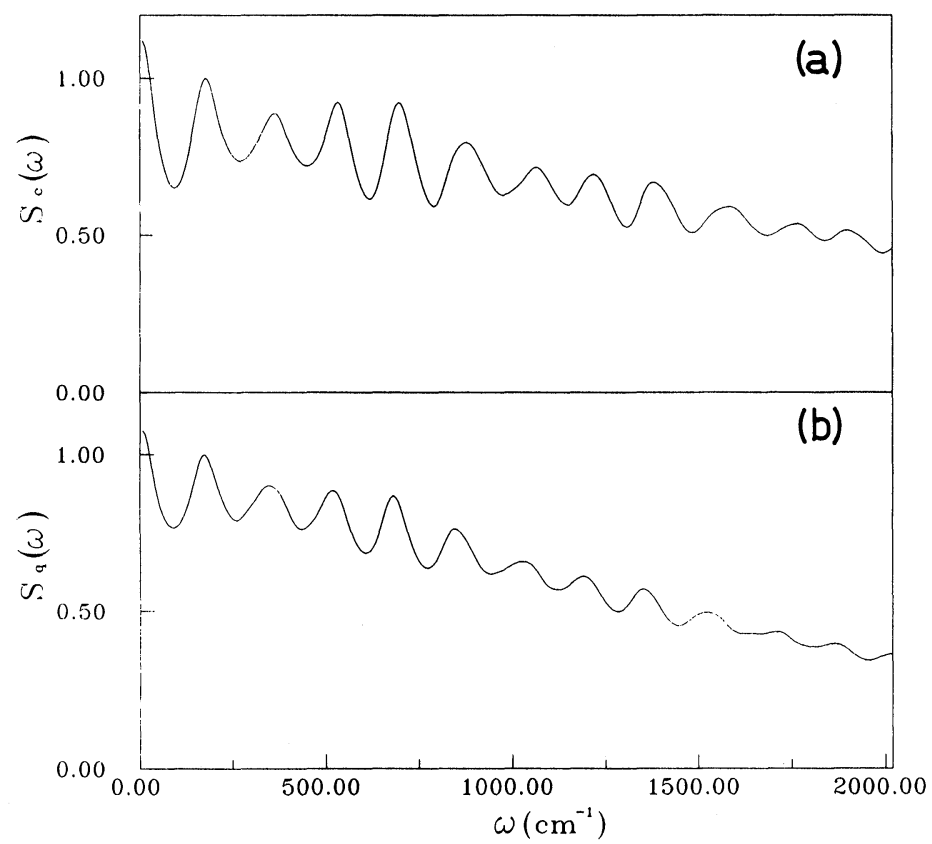

Figure 7 (a) Classical and (b) quantum low resolution spectral densities associated to the stick spectrum of Figure 5.

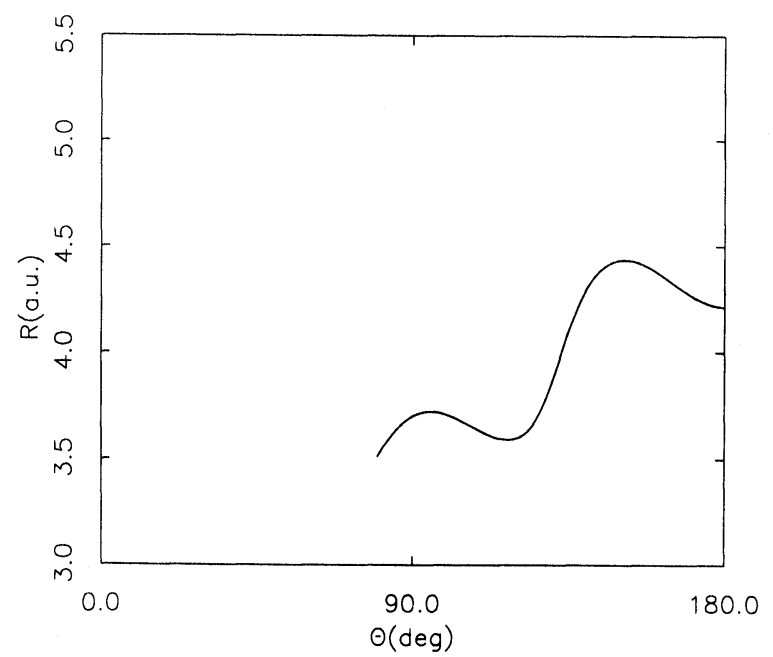

Figure 8 A representative periodic orbit of the $4: 1$ resonance ( $8: 1$ if symmetry is not taken into account) responsible for the characteristics exhibited by $C(t)$ in Figure 7 . 
trajectories with bigger contributions to the $t \approx 190 \mathrm{fs}$ peak in the correlation functions (Figure 6). The corresponding (stable) periodic orbit for the $4: 1$ resonance condiction $(8: 1$ if symmetry is not taken into account) has been calculated, and is presented in Figure 8.

As discussed in Section 3, an alternative method to obtain dynamical information of vibrationally excited molecular systems is the calculation of probability densities in phase space, such as the Husimi function. Let us now examine which relation exists between these phase space distribution functions and the structure pointed out in the quantum-classical analysis of the SEP type spectrum that we calculated for the LiCN molecule.

For that purpose we have considered classical and quantum (Husimi function) PSOS for this system, using the minimum energy path, $R_{e}(\theta)$, connecting the two stable isomers $\mathrm{LiNC} / \mathrm{LiCN}$, as sectioning plane. Proceeding in this way, we obtain PSOS's with great physical insight of the dynamics of an isomerizing system, such as ours. To define this PSOS is necessary to make the following canonical transformation

$$
\begin{gathered}
\psi=\theta \\
\rho=R-R_{e}(\theta) \\
P_{\psi}=P_{\theta}+\left(\frac{\partial R_{e}}{\partial \theta}\right)_{\theta=\psi} P_{R} \\
P_{\rho}=P_{R}
\end{gathered}
$$

Then, we have considered the $\left(\psi, P_{\psi}\right)$ quantum PSOS (see Ref. 42 for details) for the states with bigger contributions in the stick spectrum $I(E)$ of Figure 5; all of them show qualitatively the same pattern. We have represented in Figure 9 the quantum PSOS corresponding to the 25th state, the one with the biggest contribution to $I(E)$; we have also included in this figure, for the sake of comparison, the classical PSOS computed at the same (quantum) energy. Some comments are in order. In first place, the quantum phase space density appears very localized in small regions of phase space embedded in an area which is completely chaotic from the classical point of view. In second place, this localization is clearly influenced by a classical structure, namely a chain of islands of stability. Finally, and more important for the purposes of the present paper, the periodic orbit which is at the center of these islands turns out to be the same one (Figure 8) influencing the low resolution version of the spectrum presented in Figure 5. Moreover, when this periodic orbit is compared to the wavefunction of the $n=25$ state of LiCN (see Figure 10), it follows remarkably well the maxima of the wavefunction. Then, this classical element greatly influences the shape of the wavefunction, representing a sort of backbone for it. These two results constitute a strong indication that there may exist a relation between the two methods of analyzing the dynamics of highly excited molecular vibrations that we have described in this paper. This possibility deserves further investigation, and such study is currently under way. 

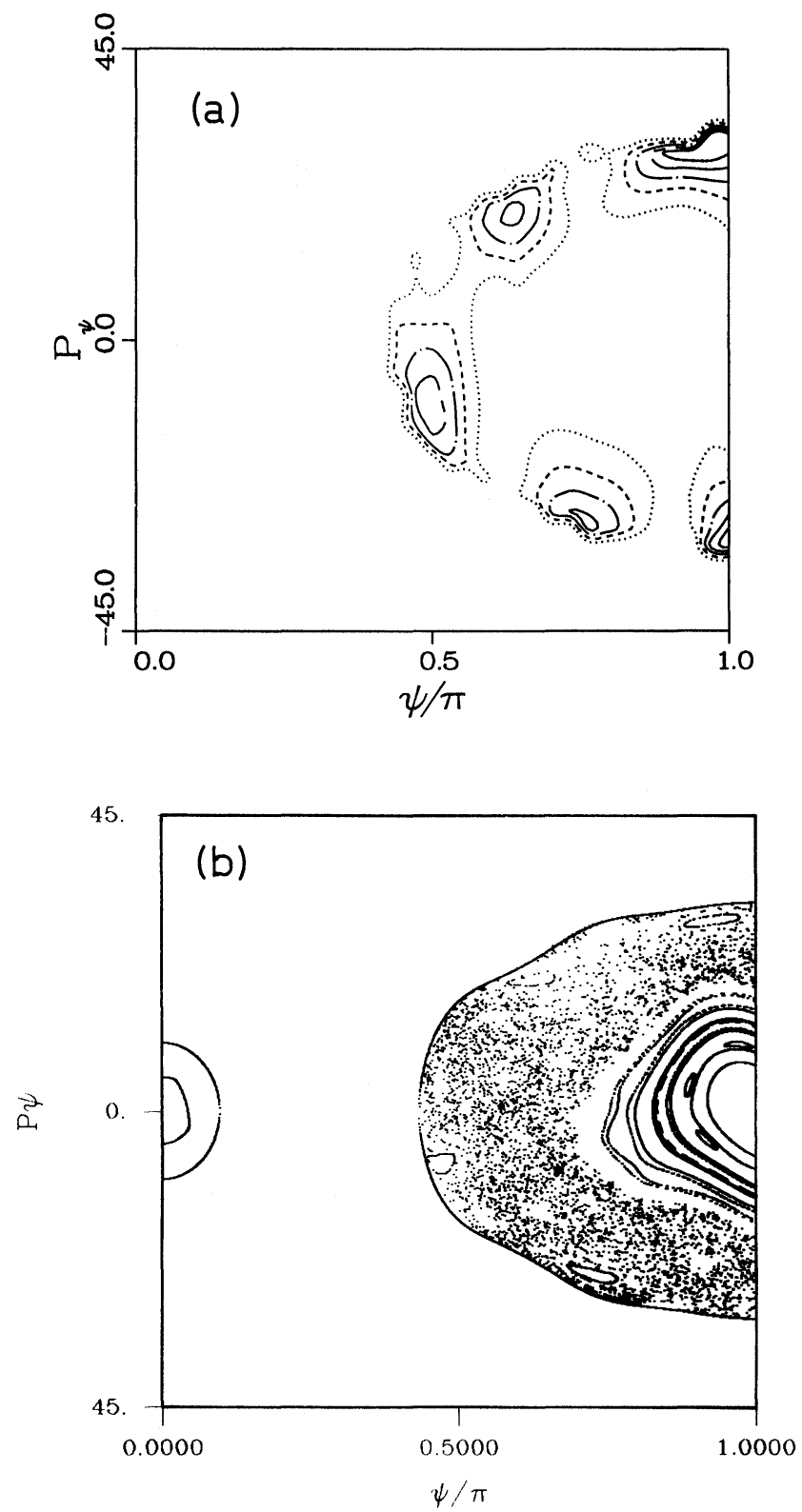

Figure 9 (a) Husimi function, and (b) classical Poincare surface of section for the 25th LiNC/LiCN state. This state corresponds to the highest peak in the spectrum of Figure 5. 


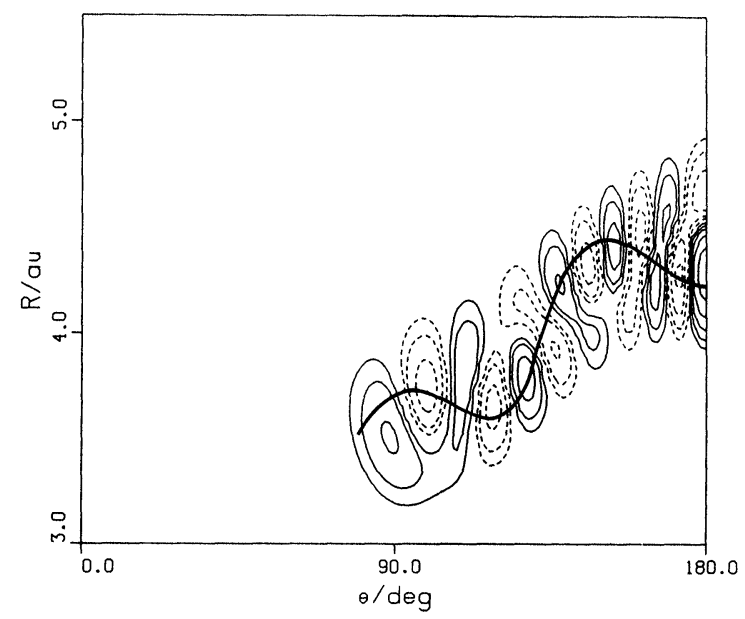

Figure 10 Periodic orbit of Figure 8 superimposed to the wavefunction of the 25 th $\mathrm{LiNC} / \mathrm{LiCN}$ state.

\section{Acknowledgements}

This work has been supported by the DGICYT (Spain) under contract PB89-117.

\section{References}

1. T. Uzer, Phys. Rep. 199, 75 (1991), and references therein.

2. R. D. Levine and R. B. Bernstein, "Molecular Reaction Dynamics and Chemical Reactivity", (Oxford U. Press, Oxford, 1974), Chap. 7.

3. V. S. Letokhov (ed.), "Laser Spectroscopy of Highly Vibrationally Excited Molecules", (Adams Hilger, New York, 1989).

4. See the special issue on SEP spectroscopy; J. Opt. Soc. Am. B7 (1990).

5. R. B. Metz, T. Kitsopoulos, A. Weaver and D. M. Neumark, J. Chem. Phys. 88, 1463 (1988); S. E. Bratforth, A. Weaver, D. W. Arnold, R. B. Metz and D. M. Neumark, J. Chem. Phys. 92, 7205 (1990).

6. A. Carrington and R. A. Kennedy, J. Chem. Phys. 81, 91 (1984).

7. E. Abranson, R. W. Field, D. Imre, K. K. Innes and J. L. Kinsey, J. Chem. Phys. 83, 453 (1985); J. P. Pique, Y. Chen, R. W. Field and J. L. Kinsey, Phys. Rev. Lett. 58, 4715 (1987).

8. M. Broyer, G. Delacretaz, G.-Q. Ni, R. L. Whetten, J.-P. Wolf and L. Woste, Phys. Rev. Lett. 62, 2100 (1989).

9. B. R. Johnson and J. L. Kinsey, Phys. Rev. Lett. 62, 1607 (1989); J. Chem. Phys. 91, 7638 (1989).

10. X. Yang, C. A. Rogaski and A. M. Wodtke, J. Opt. Soc. Am. B7, 1835 (1990).

11. O. Bohigas, M. J. Giannoni and C. Schmit, in "Quantum Chaos and Nuclear Statistical Physics", T. H. Seligman and H. Nishioka (eds.), Vol. 263 of Lectures Notes in Physics (Springer-Verlag, Berlin, 1986), p. 18.

12. B. Eckhardt, Phys. Rep. 163, 207 (1988), and references therein.

13. See for example the special issue on Molecular Vibrations: Comp. Phys. Comm. 51 (1988).

14. J. M. Gomez Llorente and E. Pollak, J. Chem. Phys. 89, 1195 (1988); 90, 5406 (1989).

15. E. J. Heller, Phys. Rev. Lett. 53, 1515 (1984); P. O'Connor, J. Gehlen and E. J. Heller, Phys. Rev. Lett. 58, 1296 (1987).

16. H. S. Taylor and J. Zakrzewski, Phys. Rev. A38, 3732 (1988).

17. J. M. Gomez Llorente, J. Zakrzewski, H. S. Taylor and K. C. Kulander, J. Chem. Phys. 89, 5959 (1988); 90, 1505 (1989). 
18. H. Goldstein, "Classical Mechanics", (Addison-Wesley, Reading, Mass., 1980).

19. I. C. Percival, Adv. Chem. Phys. 36, 1 (1977).

20. D. W. Noid and R. A. Marcus, J. Chem. Phys. 62, 2119 (1975); 67, 559 (1977).

21. S. Chapman, B. C. Garrett and W. H. Miller, J. Chem. Phys. 64, 502 (1976).

22. J. B. Swimm and R. T. Delos, J. Chem. Phys. 71, 1706 (1979); C. Jaffe and W. P. Reinhardt, J. Chem. Phys. 71, 1862 (1979); L. E. Fried and G. S. Ezra, Comp. Phys. Comm. 51, 103 (1988); D. Farrely, J. Chem. Phys. 85, 2119 (1986).

23. I. C. Percival, J. Phys. A7, 794 (1974).

24. D. W. Noid, M. L. Koszykowski and R. A. Marcus, J. Chem. Phys. 67, 404 (1977); C. Eaker, G. C. Schatz, N. De Leon and E. J. Heller, J. Chem. Phys. 81, 5913 (1984); C. C. Martens and G. S. Ezra, J. Chem. Phys. 83, 2990 (1985).

25. P. Ehrenfest, in "Sources in Quantum Mechanics", B. L. van der Waerden (ed.), (Dover, New York, 1967), p. 79.

26. E. A. Solov'ev, Sov. Phys. JETP 48, 635 (1978).

27. R. T. Skodje, F. Borondo and W. P. Reinhardt, J. Chem. Phys. 82, 4611 (1985).

28. R. T. Skodje and J. C. Cary, Comp. Phys. Rep. 8, 221 (1988); W. P. Reinhardt, Adv. Chem. Phys. 23, 925 (1989); F. Borondo, in "Structure, Interactions and Reactivity", S. Fraga (ed.), (Elsevier, Amsterdam, in press).

29. R. T. Skodje and F. Borondo, Chem. Phys. Lett. 118, 409 (1985).

30. R. T. Skodje and F. Borondo, J. Chem. Phys. 84, 1533 (1986).

31. R. T. Skodje and F. Borondo, J. Chem. Phys. 85, 2760 (1986).

32. E. Pollak, in "Theory of Chemical Reactions Dynamics", M. Baer (ed.), (CRC Press, Boca Raton, 1985).

33. A. M. Ozorio de Almeida, "Hamiltonian Systems. Chaos and Quantization", (Cambridge U. Press, New York, 1988).

34. D. Wintgen, Phys. Rev. Lett. 61, 1803 (1988).

35. J. M. Gomez Llorente, H. S. Taylor and E. Pollak, Phys. Rev. Lett. 62, 2096 (1989).

36. J. M. Gomez Llorente and H. S. Taylor, J. Chem. Phys. 91,953 (1989).

37. M. J. Davis, J. Phys. Chem. 92,3124 (1988).

38. M. C. Gutzwiller, J. Math. Phys. 12, 343 (1971).

39. S. C. Farantos, J. M. Gomez Llorente, O. Hahn and H. S. Taylor, Chem. Phys. Lett. 166, 71 (1990).

40. S. C. Farantos, J. M. Gomez Llorente, O. Hahn and H. S. Taylor, J. Chem. Phys. 93, 76 (1990).

41. S. C. Farantos, J. M. Gomez Llorente, O. Hahn and H. S. Taylor, J. Chem. Phys. 94, 2376 (1991).

42. R. M. Benito, F. Borondo, J.-H. Kim, B. G. Sumpter and G. S. Ezra, Chem. Phys. Lett. 161, 60 (1989).

43. J. R. Henderson, S. Miller and J. Tennyson, J. Chem. Soc. Faraday Trans. 86, 1963 (1990).

44. J. N. Murrell, S. Carter and L. O. Halonen, J. Mol. Spectrosc. 93, 307 (1982).

45. G. Brocks, J. Tennyson and A. van der Avoird, J. Chem. Phys. 80, 3223 (1984).

46. J. M. Gomez Llorente and E. Pollak, Chem. Phys. Lett. 138, 125 (1987).

47. J. M. Gomez Llorente, O. Hahn and H. S. Taylor, J. Chem. Phys. 92, 2762 (1990).

48. O. Hahn, J. M. Gomez Llorente and H. S. Taylor, J. Chem. Phys. 94, 2608 (1991).

49. A. P. Baronavski, Chem. Phys. Lett. 61, 532 (1979).

50. A. M. Smith, S. L. Coy, W. Klemperer and K. K. Lehmann, J. Mol. Spectrosc. 134, 134 (1989).

51. J. A. Bentley, J.-P. Brunet, R. E. Wyatt, R. A. Friesner and C. Leforestier, Chem. Phys. Lett. 161, 393 (1989).

52. M. Founargiotakis, S. C. Farantos and J. Tennyson, J. Chem. Phys. 88, 1598 (1988); S. C. Farantos and M. Founargiotakis, Chem. Phys. 142, 345 (1990). 